\title{
COMPARAÇÃO DE ÍNDICES DE SELEÇÃO NÃO PARAMÉTRICOS PARA A SELEÇÃO DE CULTIVARES ${ }^{(1)}$
}

\author{
ANTONIO AUGUSTO FRANCO GARCIA ${ }^{(2,5)} \&$ CLÁUDIO LOPES DE SOUZA JÚNIOR ${ }^{(3,4)}$
}

\begin{abstract}
RESUMO
Índices de seleção normalmente são combinações lineares que permitem a seleção para vários caracteres simultaneamente, promovendo o melhoramento das populações para o seu conjunto de características, sendo adequados a programas de seleção recorrente. Contudo, existem outros tipos de índices não lineares, também conhecidos como não paramétricos, que, por não precisarem de estimativas de parâmetros genéticos e fenotípicos, têm seu uso proposto também para a simples classificação dos indivíduos, não necessariamente em programas de seleção recorrente. Os mais comuns são o índice multiplicativo, o de soma de classificação e o de distância ao ideótipo. O presente trabalho tem por objetivo verificar se esses índices não paramétricos são adequados à seleção de cultivares, ou de genótipos em fase final de experimentação. Para tanto, utilizaram-se os resultados do Ensaio Nacional de Milho Precoce 1996/97, da EMBRAPA. Obtiveram-se as médias ajustadas para 49 híbridos, avaliados em nove locais, para os caracteres produção de espigas (kg.ha-1), umidade $(\%)$, número de dias para florescimento, altura da planta $(\mathrm{cm})$, altura da espiga $(\mathrm{cm})$, prolificidade e porcentagem de plantas acamadas e quebradas. Foram calculados parâmetros de estabilidade e adaptabilidade, também incluídos nos índices. Os resultados mostraram que os índices revistos não são adequados à seleção de cultivares, por não priorizarem os caracteres mais importantes, não permitirem o descarte dos genótipos com níveis inferiores para algumas variáveis e não preverem a realização de testes de médias.
\end{abstract}

Termos de indexação: índices de seleção, seleção de cultivares, índices não paramétricos, índices não lineares.

(1) Recebido para publicação em 29 de outubro de 1998 e aceito em 20 de maio de 1999.

(2) Departamento de Ciências Exatas, Escola Superior de Agricultura "Luiz de Queiroz" (ESALQ), Universidade de São Paulo (USP), Caixa Postal 9, 13418-900 Piracicaba (SP).

(3) Departamento de Genética, ESALQ/USP, Caixa Postal 83, 13400-970 Piracicaba (SP).

(4) Com bolsa de produtividade em pesquisa do CNPq.

(5) Com bolsa de doutorado do $\mathrm{CNPq}$ 


\title{
ABSTRACT \\ COMPARISON OF NON-PARAMETRIC SELECTION INDICES FOR THE SELECTION OF CULTIVARS
}

\begin{abstract}
Selection indices are, usually, linear combinations which allow simultaneous selection from several characters, being quite proper for recurrent selection schemes. Other nonlinear-indices (multiplicative, sum of classification and distance to the ideotype) are also available, which do not depend on genetic or phenotypic trait estimates but might be used for genotype ranking. The main aim of this research work has been to establish the feasibility of using those non-linear indices in the end of a breeding program (selection of new cultivars). Data of 49 hybrids from Brazilian Early-Flowering Maize Trials (1996/97), carried out at nine locations, have ben utilized (adjusted means for the variables ear weiht, grain humidity per cent, days-to-flowering, plant height, ear height and percentage of broken and lodged plants). Stability and adaptability parameters have been calculated as well. Results have shown that the indices studied are not suitable for cultivar selection due to the following facts: a) they do not priorize the most important traits; b) do not allow the discarding of low value genotypes and c) cannot be used for multiple comparisons.
\end{abstract}

Index terms: selection indices, cultivar selection, non-parametric indices, non-linear indices.

\section{INTRODUÇÃO}

Nos programas de melhoramento genético, freqüentemente, obtêm-se medidas de diversos caracteres com a finalidade de selecioná-los simultaneamente. Abordagens matemáticas dessa situação levaram ao desenvolvimento dos índices de seleção, que, normalmente, são obtidos como combinações lineares das medidas fenotípicas dos diversos caracteres, permitindo utilizar um único valor para efetuar a seleção de maneira mais eficiente. A idéia inicial da aplicação de índices de seleção foi apresentada por Smith (1936), com base em trabalho de Fisher (1936): conhecido como índice otimizado, sofreu, posteriormente, modificações, a maioria delas também baseada na obtenção de combinações lineares de valores fenotípicos observados. Os mais comuns são os de Brim et al. (1959), Kempthorne \& Nordskog (1959), Pesek \& Baker (1969), Tai (1977) e Smith et al. (1981).

Empregam-se tais índices para obter valores agregados de maior confiabilidade, de forma que, usando-os, obtenha-se melhoria do valor genotípico populacional. Esse valor é definido como uma função linear de valores genotípicos de diversos caracteres, que não são observáveis, ponderados por um valor econômico relativo determinado pelo melhorista. A maioria dos índices é obtida a partir da maximização da correlação entre o valor genotípico e o índice, visando à máxima eficiência na seleção: aquela que tem os índices estabelecidos como critério visa, sobretudo, melhorar gradativamente as freqüências gênicas de alelos favoráveis para o conjunto de caracteres de importância. Assim, podem ser usados nos programas de seleção recorrente, permitindo a obtenção de populações com melhor valor genotípico. Como o material selecionado será recombinado, genótipos com dados caracteres em níveis inferiores aos aceitáveis comercialmente podem ser escolhidos, desde que concentrem um número razoável de alelos favoráveis a outras características.

Outra situação encontrada é aquela em que se realizam experimentos em etapas avançadas dos programas de melhoramento genético, avaliando 
genótipos promissores (pré-comerciais), utilizando-se, normalmente, cultivares comerciais como testemunhas. Nesse caso, há necessidade de comparar os novos cultivares com os materiais comerciais existentes no mercado, baseando-se em médias obtidas a partir de experimentos em vários locais e anos. Após sua realização, os melhores materiais poderão ser liberados comercialmente. Atualmente, porém, há escassos relatos de como combinar a informação dos diversos caracteres num único valor, ou seja, num índice de seleção, o que facilitaria a comparação e a classificação dos indivíduos com base em tal conjunto de caracteres. Isso não pode ser feito pelo uso dos índices idealizados para emprego em programas de seleção recorrente, já que se deseja simplesmente identificar os melhores genótipos para o conjunto de características, e não promover o aumento do valor genotípico de uma população. Não haverá recombinação dos materiais superiores, como na seleção recorrente, e os indivíduos selecionados devem estar dentro dos padrões comerciais mínimos exigidos para a espécie para todos os caracteres. Portanto, os métodos para a obtenção de índices cujo emprego vise à melhoria do valor genotípico populacional, não são adequados a tal situação.

Alguns autores apresentaram índices não lineares, também conhecidos como índices não paramétricos, que não têm como objetivo a melhoria do valor genotípico, mas, sim, a simples classificação dos genótipos. Elston (1963) propôs um índice multiplicativo que considera todos os caracteres com o mesmo peso econômico. Em 1972, Schwarzbach, citado por Wricke \& Weber (1986), sugeriu o uso de medidas de distância, tais como a euclidiana e a de Mahalanobis, para classificar os genótipos para várias características, simultaneamente, em função de sua proximidade com um genótipo ideal, definido pelo pesquisador. Mulamba \& Mock (1978) desenvolveram um índice no qual se efetua a soma do número de ordem que o genótipo apresenta para cada caráter mensurado; quanto menor o valor obtido, melhor a classificação. Em todos esses casos, não são necessárias estimativas de parâmetros genéticos, como para os índices lineares. Nesse contexto, o presente trabalho tem por objetivo comparar esses três índices não paramétricos quanto a sua validade para a seleção de cultivares, ou seja, genótipos em etapas finais de avaliação, por meio de um caso prático.

\section{MATERIAL E MÉTODOS}

Utilizaram-se os resultados do Ensaio Nacional de Cultivares de Milho Precoce, referente ao ano agrícola 1996/97, para verificar a adequação dos três índices a uma situação real. Deve-se ressaltar que, na prática, o número de genótipos pré-comerciais avaliados nas etapas finais dos programas de desenvolvimento de cultivares deve ser muito maior que o encontrado em tais experimentos. Esse ensaio é feito anualmente, em diversas localidades, sob coordenação da Empresa Brasileira de Pesquisa Agropecuária (EMBRAPA), mediante o Centro Nacional de Pesquisa de Milho e Sorgo, localizado em Sete Lagoas (MG). Tomaram-se resultados de dez locais, escolhidos em diferentes regiões do País, a saber: Ataliba Leonel (SP), Brasília (DF), Campo Alegre (MS), Capinópolis (SP), Cascavel (PR), Goianésia (GO), Rondonópolis (MT), São Gabriel do Oeste (MS), Sooretama (ES) e Tangará da Serra (GO). Nem sempre os caracteres avaliados foram medidos em todos os locais. Nos experimentos, instalados segundo o delineamento látice quadrado 7 x 7 , avaliaram-se 49 híbridos.

As parcelas constituíram-se de duas linhas de $5 \mathrm{~m}$, espaçadas entre si por $0,90 \mathrm{~m}$, sendo todos os experimentos com três repetições. Em cada uma das parcelas dos respectivos locais, estimaram-se os seguintes caracteres: produção de espigas (PE, kg.ha-1), umidade nos grãos (U, \%); número de dias para florescimento (FLO); altura da planta (AP, cm) e da espiga (AE, cm); número de espigas e de plantas por parcela, cuja razão originou a variável prolificidade (PROL); número de plantas acamadas e quebradas (PAQ, \%), que, somados, divididos pelo estande e multiplicados por 100, resultaram no número de plantas acamadas e quebradas em porcentagem. 
Em cada local, realizaram-se as análises da variância de acordo com o modelo matemático do delineamento látice quadrado (Cochran \& Cox, 1957). Foram estimadas as médias ajustadas de cada tratamento, em cada experimento, para a variável PE, as quais foram usadas, posteriormente, para a análise de estabilidade desse caráter. As análises foram efetuadas com os dados em suas unidades originais, exceto para a variável PAQ, transformada pelo uso da função $f(x)=\sqrt{x+0,5}$. Realizou-se, em seguida, a análise da variância conjunta dos respectivos locais para todos os caracteres. Os efeitos, com exceção do erro experimental, foram considerados como fixos. Para a variável PE, foi incluída no modelo a covariável estande, de forma que a produção fosse também ajustada para esse fator (Steel \& Torrie, 1960). Em seguida, estimaram-se as médias ajustadas, pelo método dos quadrados mínimos, para todas as variáveis. A partir dos resíduos intrablocos obtidos nas análises da variância conjunta, calcularam-se os erros efetivos (Cochran \& Cox, 1957). Realizaram-se as análises estatísticas mencionadas, utilizando-se o programa computacional SAS (SAS INSTITUTE INC., 1992), adotando o procedimento conhecido como GLM (General Linear Models). Efetuou-se a análise de estabilidade para a variável PE envolvendo os nove locais onde ela foi avaliada, pelo método de Eberhart \& Russell (1966). Obtiveram-se, assim, as estimativas do parâmetro $b$ (adaptabilidade), coeficiente angular da reta de regressão entre o desempenho de cada genótipo e os índices de ambientes. Tais índices foram tomados como sendo as diferenças entre a médias dos experimentos nos respectivos locais e a média de todos os experimentos. Além disso, calcularam-se os ajustes à regressão linear, pelo coeficiente de determinação ( $\mathrm{R}^{2}$, estabilidade).

\section{1 Índice multiplicativo}

Aplicou-se esse índice, proposto por Elston (1963), às médias ajustadas das análises da variância conjuntas dos caracteres PE, U, FLO, AP, AE, PROL e PAQ, e também as estimativas do parâmetro de estabilidade $\left(\mathrm{R}^{2}\right)$. Não foi possível aplicá-lo às estimativas de $b$, uma vez que o índice só permite a otimização da seleção de indivíduos extremos (superiores ou inferiores), e, nesse caso, o valor ideal da variável é 1,0, intermediário. Para nenhuma das variáveis se supôs que algum dos híbridos com valor nos níveis mínimos ou máximos fosse descartado; para tanto, em vez de subtrair de cada resultado a maior ou menor ocorrência da variável, foram subtraídos valores de $k_{i}$ de modo que não surgissem resultados iguais a zero:

$\left\{k_{i}=[n(\min P i)-(\max P i)] /[n-1]\right\}$, obtido com base na distribuição teórica de uma amostra aleatória com distribuição retangular (Elston, 1963). Foram seguidos os passos abaixo:

i) Tomaram-se escalas para os diferentes caracteres, de forma que os melhores híbridos sempre possuíssem os maiores valores. Para tanto, obtiveram-se os recíprocos das medidas dos caracteres U, FLO, AP, AE e PAQ. Com tal tranformação, esses caracteres passaram a apresentar resultados menores que a unidade, o que já ocorreu originalmente para PROL e $\mathrm{R}^{2}$. Como números menores que 1,0 apresentam logaritmos negativos, multiplicou-se cada variável com valor menor que a unidade por uma constante que elevasse a escala de mensuração do respectivo caráter a valores maiores que 1,0. Após as multiplicações, os caracteres obtidos foram: PE, (1/U) $\mathrm{x}$ $10^{2},(1 / \mathrm{FLO}) \times 10^{2},(1 / \mathrm{AP}) \times 10^{3},(1 / \mathrm{AE}) \times 10^{3}, \mathrm{PROL}$ x $10,(1 / \mathrm{PAQ}) \mathrm{x} 10, \mathrm{R}^{2}$ x 10 .

ii) Aplicou-se a transformação logarítmica a todas essas $i$ variáveis, visando tornar as distribuições uniformes. Em outras palavras, calculou-se $P_{i}^{\prime}$, o logaritmo de cada variável $i$.

iii) Para cada $p_{i}{ }^{\prime}$, subtraiu-se $k_{i}^{\prime}=\left[n\left(\min p^{\prime} i\right)\right.$ - (máx $\left.\left.p^{\prime} i\right)\right] /[n-1]$, obtendo-se $\left(p_{i}{ }^{\prime}-k_{i}{ }^{\prime}\right)$, onde $n$ é o número de híbridos. Com essa subtração, eliminou-se a diferença de escala entre as variáveis.

iv) Como os histogramas obtidos para os caracteres não apresentaram formas similares, calculou-se $p_{i}{ }^{\prime \prime}=\log \left[\left(p_{i}{ }^{\prime}-k_{i}{ }^{\prime}\right) \cdot 10^{4}\right]$. A constante $10^{4}$ foi usada para evitar logaritmos negativos para $p_{i}{ }^{\prime \prime}$.

v) Subtraiu-se $k_{i}{ }^{\prime \prime}=\left[n\left(\min p_{i}{ }^{\prime \prime}\right)-\left(\max p_{i}{ }^{\prime \prime}\right)\right] /$ [n-1] de cada $P_{i}^{\prime \prime}$, resultando em $\left(p_{i}{ }^{\prime \prime}-k_{i}^{\prime \prime}\right)$. As distri- 
buições foram comparadas por meio de histogramas e se mostraram semelhantes, não necessitando nova aplicação da transformação.

vi) Calcularam-se os índices multiplicativos $\left[I_{m}=\prod_{i=1}^{7}\left(p_{i}{ }^{\prime \prime}-k_{i}{ }^{\prime \prime}\right)\right]$ para os 49 híbridos, efetuando-se sua classificação com base nos resultados obtidos, sendo os melhores aqueles que apresentaram os maiores valores.

\section{2 Índice de soma de classificação}

Para esse índice, proposto por Mulamba \& Mock (1978), classificaram-se os híbridos para os caracteres PE, U, FLO, AP, AE, PROL, PAQ e R², recebendo todo indivíduo um número de classificação para cada caráter, adotando-se o critério de sempre utilizar número de classificação 1 para o melhor valor do respectivo caráter e, assim, sucessivamente. Desse modo, os indivíduos com classificação 1 foram aqueles com maiores médias para os caracteres PE, PROL e $\mathrm{R}^{2}$ e, os com menor valor, para U, FLO, AP, AE e PAQ. Não foi possível incluir a variável $b$, já que ela não deve ser maximizada ou minimizada, como prevê o índice, mas, sim, permitir a melhor classificação dos híbridos com a estimativa mais próxima possível da unidade. Após obtenção dos números de classificação de cada genótipo, calcularam-se seus índices da seguinte forma:

$$
I_{j}=\Sigma n_{i j}
$$

sendo

$I_{j}=$ índice para o genótipo $j$;

$n_{i j}=$ número de classificação do caráter $i$ para o genótipo $j$.

Foram considerados melhores os híbridos com menores $I_{j} s$.

\section{3 Índice de distância ao ideótipo}

Para obtenção desse índice, Schwarzbach, 1972, citado por Wricke \& Weber, 1986), calcularam-se, a partir das médias fenotípicas ajustadas, as distâncias de cada indivíduo a um genótipo ideal, definido para os caracteres PE, U, FLO, AP, AE, PROL, $\mathrm{PAQ}, b$ e $\mathrm{R}^{2}$. Inicialmente, essas variáveis foram estandardizadas. Depois, definiu-se o ideótipo como sendo o genótipo formado pelo maior valor dos caracteres PE, PROL e $\mathrm{R}^{2}$, menor valor para U, FLO, AP, AE e PAQ e valor 1,0 para $b$. Em seguida, calcularam-se as distâncias euclidianas entre cada genótipo e esse ideótipo, pelo uso da fórmula:

$$
d_{j I}=\sqrt{\sum_{i=1}^{9}\left(x_{i j}-x_{I i}\right)^{2}}
$$

em que

$d_{j I}=$ distância euclidiana entre o genótipo $j$ e o ideótipo $I(j=1, \ldots, 49)$;

$x_{i j}=$ medida do caráter $i$ no genótipo $j(i=1, \ldots, 9)$;

$x_{I i}=$ valor definido para o ideótipo $I$, referente ao caráter $i$.

Procedeu-se, então, à classificação dos indivíduos com base na distância que apresentaram do ideótipo, sendo considerados melhores aqueles cujas distâncias foram as menores.

\section{RESULTADOS E DISCUSSÃO}

\section{1 Índice multiplicativo}

O índice multiplicativo não requer a obtenção de estimativas de parâmetros genéticos nem supõe a existência de um valor genotípico populacional. Isso permitiu que fosse sugerida sua aplicação com a simples finalidade de classificar os genótipos com respeito a diversos caracteres, simultaneamente, como é o caso da seleção de cultivares. Como desvantagem, impede a inclusão do parâmetro de adaptabilidade $(b)$ nos cálculos, permitindo somente a de variáveis que se deseja maximizar ou minimizar. Desse modo, outros parâmetros cuja seleção seja estabilizadora, como, por exemplo, aquela realizada para os caracteres dias para maturação, altura das plantas intermediária para possibilitar colheita mecânica, etc., também não podem ser incluídos nos cálculos. A impossibilidade de descartar valores abaixo ou acima de determinados limites é uma grande restrição, pois, na prática, os melhoristas normalmente estão interessados em des- 
cartar valores acima ou abaixo de dados limites. Seus valores variaram de 0,00 a 29,01, com média de 13,5; quanto maior o valor do índice, melhor o genótipo. Vale lembrar que os valores de $I_{m}$ são as únicas informações que o melhorista teria, originalmente, para classificar os híbridos, o que, evidentemente, impossibilita estudar o ocorrido. Para facilitar, esses valores do índice foram agrupados aos dados originais, permitindo melhor visualização de como foi feita a classificação de cada variável. O caráter PAQ foi mostrado em sua unidade original (porcentagem de plantas acamadas e quebradas em relação ao estande), embora os cálculos realizados para a obtenção do índice tenham sido feitos com essa variável transformada (Quadro 1).

Por se tratar de um índice multiplicativo, sem nenhuma possibilidade de ponderação, ocorreu que caracteres mais importantes, como PE, tivessem o mesmo peso na classificação que os demais. Na prática, os melhoristas jamais aceitariam isso, uma vez que a variável citada, pelo menos no exemplo, é mais importante que as demais. Mesmo para outras espécies, normalmente, deve haver necessidade de algum tipo de ponderação. Um exame da classificação dos indivíduos mostra que, entre os dez primeiros colocados para PE, encontram-se os híbridos com classificação 19, 6, 3, 14, 37, 20, 10, 5, 4 e 25. Obviamente, os melhoristas não aceitariam que esses materiais fossem recomendados para cultivo. Os híbridos classificados em 1. e 2. lugar para PE posicionaram-se apenas em $45 .^{\circ}$ e $24 .^{\circ}$ lugar respectivamente, o que não é esperado de um índice adequado à seleção de cultivares.

É possível definir que os híbridos com valor máximo ou mínimo para algum atributo não sejam selecionados (Elston, 1963). Na situação real, porém, é mais provável que o melhorista deseje definir valores abaixo (ou acima) dos quais os indivíduos sejam descartados. É mais comum ocorrer a imposição de faixas de variação e não apenas limites extremos. Por exemplo, pode-se desejar que os híbridos com produção estatisticamente inferior à das testemunhas comerciais sejam descartados; outro caso seria impedir a seleção de materiais com altura acima da média das variedades disponíveis no mercado, e assim por diante. Tal situação não é abordada no índice multiplicativo. Caso fosse utilizado $k_{i}$ maior que o valor mínimo, surgiriam números negativos para $P_{i}-k_{i}$, e haveria problemas na obtenção do índice e dos logaritmos. Vale lembrar que o produto de dois números negativos é positivo, o que pode causar grandes distorções na classificação.

Os valores do índice, mesmo acompanhados dos dados originais, não permitem visualizar se, no grupo de híbridos selecionados, existe algum material que se deva descartar ter apresentado desempenho inferior para alguma variável (Quadro 1). Logo, o fato de o índice não apresentar maiores subsídios para o melhorista julgar o resultado da classificação é uma grande desvantagem, visto que distorções podem ocorrer e não ser notadas. Diversos exemplos podem ser citados. O híbrido classificado em 1..$^{\circ}$ lugar com o índice, XL 345, tem PE localizado apenas no 19. lugar, com U em 18. e PAQ em 21. ' lugar; isso implica que ele jamais seja recomendado como cultivar pelos melhoristas. O híbrido C $435 \mathrm{G}$ foi classificado em 6. lugar com o índice e apresentou AE na 37. ${ }^{a}$ posição, o que possivelmente implicaria seu descarte. $\mathrm{O}$

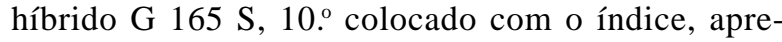
sentou FLO na 39. posição, o que poderia caracterizálo como material tardio, resultando no seu descarte. O Z 84 E 98, 2. ${ }^{\circ}$ colocado com o índice, tem U classificada em $45 .^{\circ}$, o que talvez impeça sua recomendação. Fica evidente que o simples emprego do índice dificulta ao melhorista julgar se realmente foram selecionados os prováveis cultivares. Os híbridos classificados nos cinco primeiros lugares pelo índice têm colocação para PE em $19 .^{\circ}, 6 .^{\circ}, 3 .^{\circ}, 14 .^{\circ}$ e $37 .^{\circ}$ lugar respectivamente. Isso não seria aceito em situações reais, dada a importância que esse caráter apresenta. Os híbridos mais produtivos poderiam ser descartados simplesmente por não fazer parte do grupo mais bem classificado com uso do índice, o que não deve ocorrer quando a seleção visa à obtenção de cultivares.

Possivelmente, o fato de todos os caracteres terem o mesmo peso foi a causa de a classificação dos híbridos não ter validade prática para os melhoristas, em se tratando da seleção de cultivares. 
Quadro 1. Índice multiplicativo ( $\mathrm{I}_{\mathrm{m}}$ ) para os 49 híbridos com classificação para $\mathrm{I}_{\mathrm{m}}$ e cada uma das variáveis: produção de espigas (PE), umidade (U), número de dias para florescimento (FLO), altura da planta (AP), altura da espiga (AE), prolificidade (PROL), número de plantas acamadas e quebradas (PAQ)

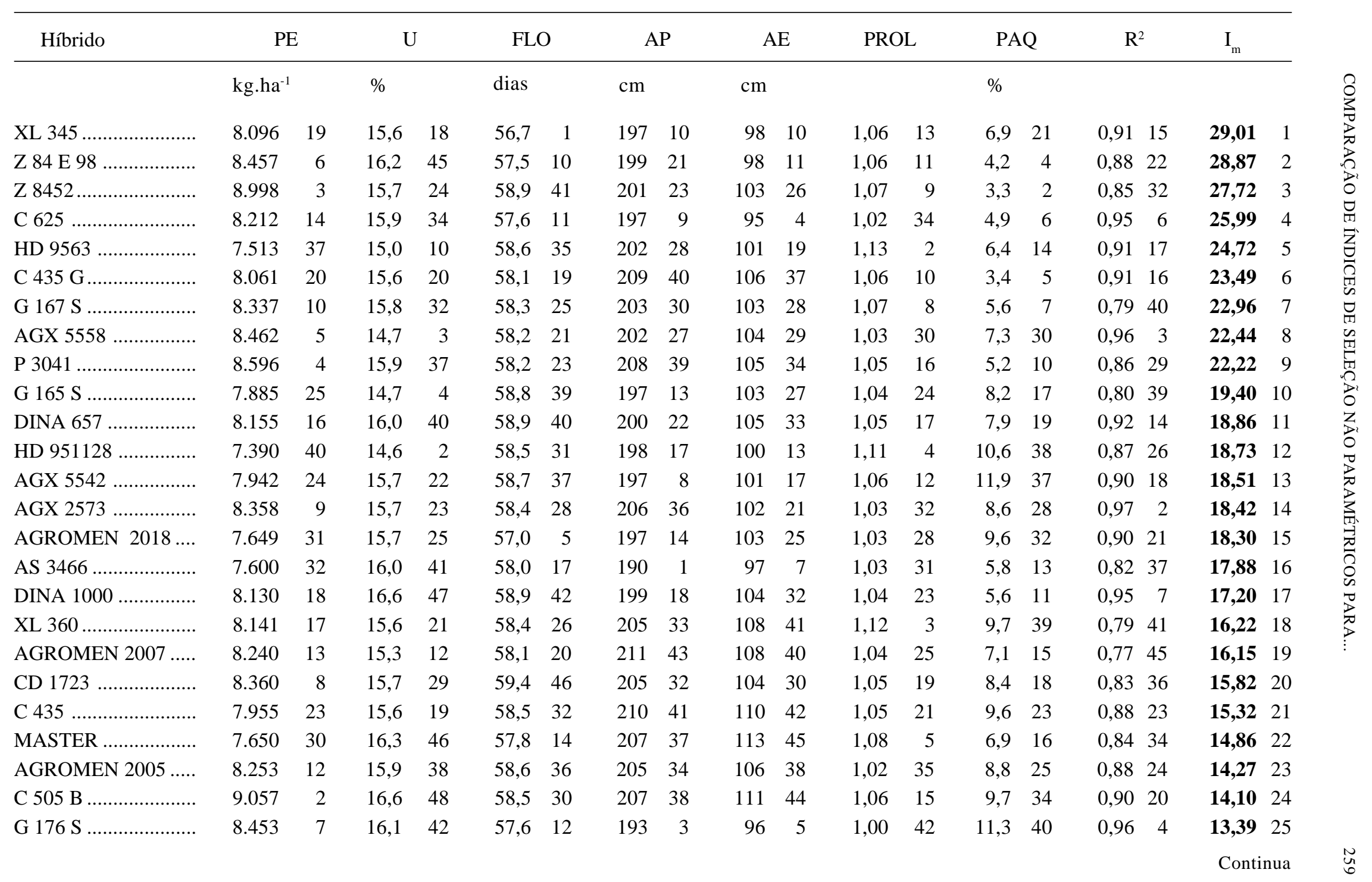


Quadro 1. Conclusão

\begin{tabular}{|c|c|c|c|c|c|c|c|c|c|c|c|c|c|c|c|c|c|}
\hline \multirow[t]{2}{*}{ Híbrido } & \multicolumn{2}{|c|}{$\mathrm{PE}$} & \multicolumn{2}{|c|}{$\mathrm{U}$} & \multicolumn{2}{|c|}{ FLO } & \multicolumn{2}{|c|}{$\mathrm{AP}$} & \multicolumn{2}{|c|}{$\mathrm{AE}$} & \multicolumn{2}{|c|}{ PROL } & \multicolumn{2}{|c|}{ PAQ } & $\mathrm{R}^{2}$ & \multicolumn{2}{|l|}{$\mathrm{I}_{\mathrm{m}}$} \\
\hline & kg.ha- ${ }^{-1}$ & & $\%$ & & dias & & $\mathrm{cm}$ & & $\mathrm{cm}$ & & & & $\%$ & & & & \\
\hline EXCELLER & 7.544 & 35 & 15,9 & 33 & 58,7 & 38 & 210 & 42 & 105 & 35 & 1,05 & 20 & 8,4 & 22 & $0,87 \quad 25$ & 13,24 & 27 \\
\hline Z 84 E 90 & 7.430 & 39 & 15,9 & 35 & 57,4 & 9 & 194 & 4 & 94 & 2 & 1,00 & 40 & 8,4 & 24 & $0,94 \quad 9$ & 13,23 & 28 \\
\hline C $491 \mathrm{~W}$ & 7.519 & 36 & 15,9 & 36 & 58,0 & 18 & 204 & 31 & 101 & 18 & 1,08 & 6 & 15,5 & 47 & $0,93 \quad 13$ & 12,88 & 29 \\
\hline $\mathrm{CO} 34$ & 7.381 & 42 & 15,7 & 28 & 58,3 & 24 & 203 & 29 & 99 & 12 & 1,00 & 41 & 4,9 & 9 & $0,90 \quad 19$ & 12,01 & 31 \\
\hline HD 9486 & 7.858 & 26 & 15,6 & 16 & 59,1 & 43 & 211 & 44 & 107 & 39 & 1,04 & 26 & 8,6 & 33 & $0,78 \quad 42$ & 10,76 & 32 \\
\hline $\mathrm{R} \& \mathrm{G} 01$ & 7.315 & 45 & 16,0 & 39 & 59,1 & 44 & 197 & 12 & 102 & 22 & 1,03 & 27 & 9,3 & 36 & $0,94 \quad 8$ & 10,73 & 33 \\
\hline AG 5014 & 7.741 & 29 & 16,1 & 43 & 58,5 & 29 & 197 & 11 & 102 & 24 & 1,03 & 33 & 14,5 & 41 & $0,80 \quad 38$ & 10,61 & 34 \\
\hline Z 8440 & 7.264 & 47 & 15,3 & 13 & 56,9 & 4 & 193 & 2 & 91 & 1 & 1,00 & 43 & 9,5 & 35 & $0,77 \quad 43$ & 10,07 & 35 \\
\hline PL 322 & 7.313 & 46 & 15,6 & 17 & 57,6 & 8 & 216 & 46 & 114 & 46 & 1,02 & 36 & 7,2 & 20 & $0,97 \quad 1$ & 10,00 & 36 \\
\hline PL 320 & 7.335 & 43 & 15,8 & 31 & 59,2 & 45 & 221 & 48 & 114 & 47 & 1,03 & 29 & 13,7 & 42 & $0,93 \quad 12$ & 5,20 & 40 \\
\hline CO 32 & 7.756 & 28 & 15,8 & 30 & 57,8 & 13 & 201 & 24 & 100 & 16 & 0,99 & 47 & 6,4 & 12 & $0,84 \quad 33$ & 2,86 & 41 \\
\hline AGX 5492 & 7.820 & 27 & 14,8 & 5 & 58,0 & 16 & 205 & 35 & 105 & 36 & 0,99 & 48 & 8,5 & 26 & $0,87 \quad 27$ & 2,35 & 42 \\
\hline FT 9006 & 7.383 & 41 & 15,0 & 11 & 58,4 & 27 & 196 & 7 & 100 & 14 & 1,01 & 38 & 15,0 & 48 & $0,83 \quad 35$ & 1,69 & 43 \\
\hline A 953 & 6.843 & 49 & 14,5 & 1 & 56,9 & 3 & 198 & 16 & 100 & 15 & 1,06 & 14 & 4,0 & 3 & $0,77 \quad 44$ & 0,75 & 44 \\
\hline C $505 \mathrm{C}$ & 9.531 & 1 & 17,7 & 49 & 58,6 & 34 & 212 & 45 & 111 & 43 & 1,07 & 7 & 8,4 & 27 & $0,96 \quad 5$ & 0,49 & 45 \\
\hline AGROMEN 2003 & 8.321 & 11 & 15,5 & 15 & 57,9 & 15 & 201 & 26 & 101 & 20 & 0,98 & 49 & 4,9 & 8 & $0,69 \quad 48$ & 0,42 & 46 \\
\hline AL 25 XII & 7.431 & 38 & 15,0 & 7 & 60,4 & 48 & 219 & 47 & 118 & 48 & 1,04 & 22 & 15,1 & 49 & $0,86 \quad 30$ & $\mathbf{0 , 0 3}$ & 47 \\
\hline HT 951005 & 7.571 & 34 & 14,9 & 6 & 60,5 & 49 & 199 & 19 & 104 & 31 & 1,22 & 1 & 10,3 & 31 & $0,66 \quad 49$ & $\mathbf{0 , 0 1}$ & 48 \\
\hline DINA 270 & 8.164 & 15 & 15,0 & 8 & 59,9 & 47 & 230 & 49 & 127 & 49 & 1,00 & 46 & 9,1 & 29 & $0,93 \quad 11$ & $\mathbf{0 , 0 0}$ & 49 \\
\hline
\end{tabular}


O índice multiplicativo foi idealizado para selecionar, aproximadamente, os mesmos indivíduos que o linear, devendo ser adequado somente à seleção recorrente.

Outro ponto a ser levantado é sobre as reais diferenças entre as médias de cada variável, já que o índice não prevê a realização de testes de comparações múltiplas. As diferenças entre as médias fenotípicas de cada variável podem não ser estatisticamente significativas e, usando-se o índice diretamente nas médias, pode-se incorrer no erro de considerar como diferentes médias que, na verdade, são estatisticamente iguais. Isso deveria ser levado em conta na seleção de cultivares.

Finalizando, embora tenha sido recomendado também com a simples finalidade de classificar os genótipos, o índice multiplicativo mostra sérias restrições quanto ao uso nos programas de seleção de cultivares.

\section{2 Índice de soma de classificação}

Tal índice foi obtido somando os valores de classificação para cada caráter de cada genótipo (Quadro 2). Optou-se por apresentar a variável PAQ em suas unidades originais, já que a tranformação aplicada não alteraria tais valores, que variaram entre 107 e 297, com média 200.

Verifica-se que, entre os 10 primeiros colocados, estão os híbridos com valores classificados para PE nas posições 19, 14, 6, 49, 5, 7, 3, 37, 39 e 20. Tal fato jamais seria aceito pelos melhoristas, uma vez que o último colocado (49. ) para esse caráter, o híbrido A 953, está entre o grupo selecionado (4. ${ }^{\circ}$ lugar). Evidentemente, isso ocorreu porque outros seus atributos são favoráveis, como U (1. ${ }^{\circ}$ lugar), FLO ( $3 .^{\circ}$ lugar) e PAQ (3. lugar). De fato, isso é aceito em programas de seleção recorrente, onde valores desfavoráveis em alguns caracteres podem ser compensados por valores adequados em outros. Não se aceita isso, contudo, na seleção de cultivares, uma vez que os materiais devem ser recomendados para plantio e nenhum caráter pode estar fora dos níveis mínimos aceitáveis. Os cinco híbridos mais produtivos (maior PE) encontram-se nas posições $28,40,7,21$ e 5 , respectiva- mente, após o emprego do índice. O 1. ${ }^{\circ}$ e o $22^{\circ}$ colocado para PE, os híbridos C 505 C e C 505 B, nem sequer seriam recomendados como cultivares, por estarem na 28. e 40 . posição respectivamente; isso mostra que ele não pode ser usado para a seleção de cultivares.

O índice não permite descartar híbridos com caracteres em níveis inferiores, o que, praticamente, inviabiliza o seu uso, já que nas etapas finais nunca devem ser selecionados. Vale lembrar que o resultado da aplicação do índice de soma de classificação fornece apenas os valores de $I_{j}$, impedindo verificar quais os valores apresentados para as variáveis dos híbridos selecionados e descartados, o que é outra desvantagem. Os valores de cada variável foram aqui mostrados para possibilitar avaliar sua aplicação.

A utilização desse índice é muito simples, não necessitando ajustar as unidades das variáveis, como no caso do multiplicativo. Como não são usados os valores fenotípicos diretamente, e, sim, um número associado a cada um deles, é evidente que a variância é a mesma para todas as variáveis, evitando tranformações de dados. Desconhece-se, contudo, se as diferenças entre as médias são ou não significativas, o que pode levar a interpretações errôneas quanto às reais diferenças entre os híbridos. Por exemplo, os híbridos XL 345 e C 625 mostraram os mesmos valores para a variável AP $(197 \mathrm{~cm})$, mas com números de classificação diferentes, implicando valores diferentes para o índice (Quadro 2). Para outras variáveis, isso também deve ter ocorrido, sem que o melhorista tenha conhecimento sobre a significância das diferenças entre as médias. Assim, pode-se observar que esse índice não seria indicado para a seleção de cultivares.

\section{3 Índice baseado em medida de distância}

Tal índice foi calculado como a distância euclidiana entre cada híbrido e o ideótipo (Quadro 3). O fato de permitir a inclusão de variáveis cujos valores não sejam os extremos, mas, sim, qualquer valor de interesse do melhorista, uma vez que o ideótipo é que fixou, é uma vantagem que tal índice apresenta sobre o multiplicativo e o de soma de classificação, que não permitiram a inclusão de $b$. 
Quadro 2. Índice de soma de classificação $\left(I_{j}\right)$ para os 49 híbridos com classificação para $I_{j}$ e cada uma das variáveis: produção de espigas (PE), umidade (U), número de dias para florescimento (FLO), altura da planta (AP), altura da espiga (AE), prolificidade (PROL), número de plantas acamadas e quebradas (PAQ)

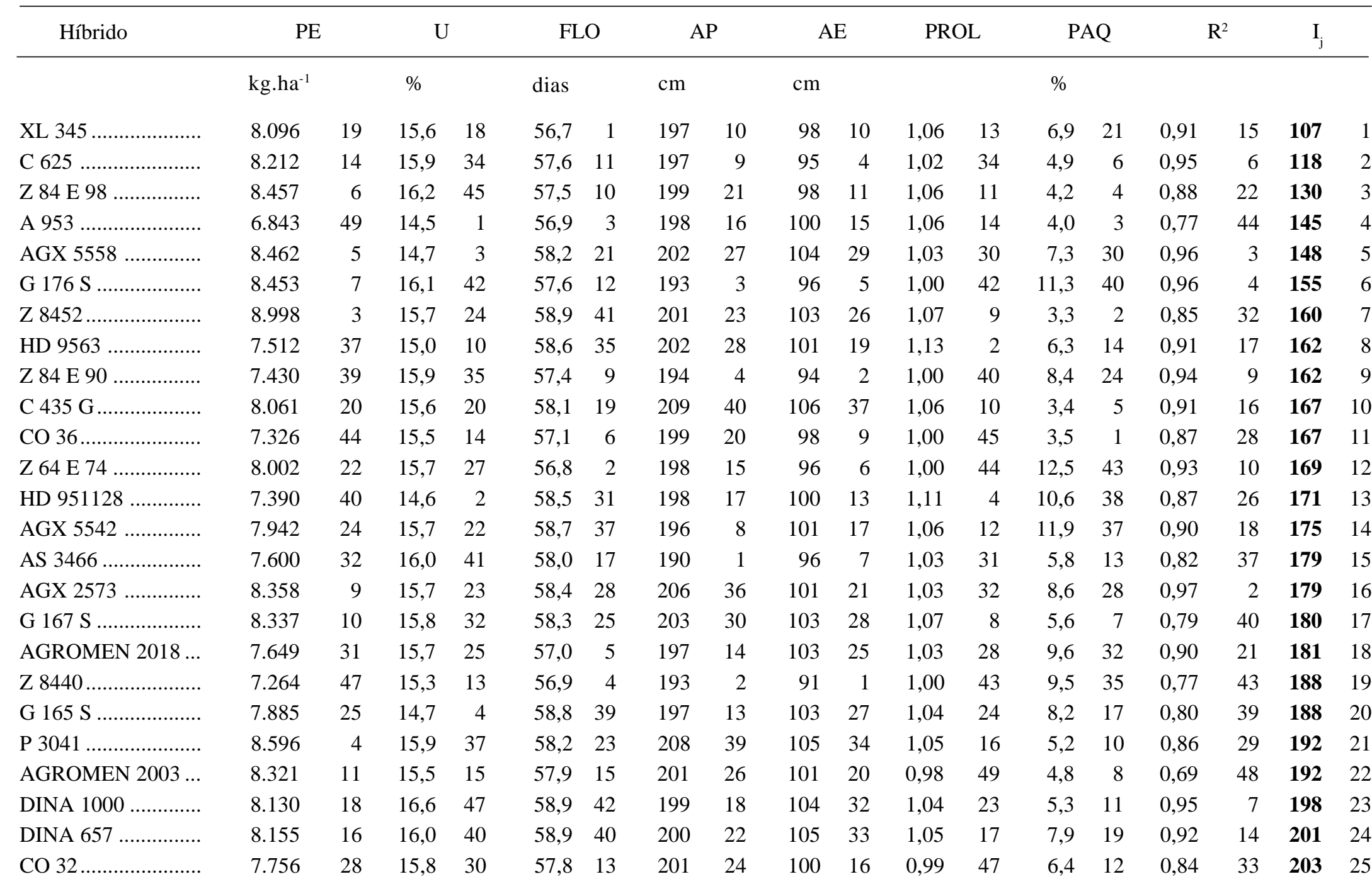

(1)


Quadro 2. Conclusão

\begin{tabular}{|c|c|c|c|c|c|c|c|c|c|c|c|c|c|c|c|c|c|c|}
\hline \multirow[t]{2}{*}{ Híbrido } & \multicolumn{2}{|c|}{$\mathrm{PE}$} & \multicolumn{2}{|c|}{$\mathrm{U}$} & \multicolumn{2}{|c|}{ FLO } & \multicolumn{2}{|c|}{$\mathrm{AP}$} & \multicolumn{2}{|c|}{$\mathrm{AE}$} & \multicolumn{2}{|c|}{ PROL } & \multicolumn{2}{|c|}{ PAQ } & \multicolumn{2}{|c|}{$\mathrm{R}^{2}$} & \multicolumn{2}{|l|}{$\mathrm{I}_{\mathrm{j}}$} \\
\hline & $\mathrm{kg} \cdot \mathrm{ha}^{-1}$ & & $\%$ & & dias & & $\mathrm{cm}$ & & $\mathrm{cm}$ & & & & $\%$ & & & & & \\
\hline C $491 \mathrm{~W}$ & 7.519 & 36 & 15,9 & 36 & 58,0 & 18 & 204 & 31 & 101 & 18 & 1,08 & 6 & 15,5 & 47 & 0,93 & 13 & 205 & 27 \\
\hline 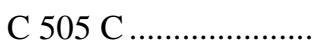 & 9.531 & 1 & 17,4 & 49 & 58,6 & 34 & 212 & 45 & 110 & 43 & 1,07 & 7 & 8,4 & 27 & 0,96 & 5 & 211 & 28 \\
\hline AGX 5674 ..................... & 8.020 & 21 & 15,7 & 26 & 58,2 & 22 & 195 & 5 & 98 & 8 & 1,02 & 37 & 14,8 & 45 & 0,70 & 47 & 211 & 29 \\
\hline 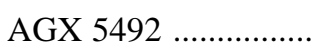 & 7.820 & 27 & 14,8 & 5 & 58,0 & 16 & 205 & 35 & 105 & 36 & 0,99 & 48 & 8,5 & 26 & 0,87 & 27 & 220 & 32 \\
\hline PL 322 & 7.313 & 46 & 15,6 & 17 & 57,4 & 8 & 216 & 46 & 114 & 46 & 1,02 & 36 & 7,2 & 20 & 0,97 & 1 & 220 & 33 \\
\hline 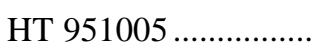 & 7.571 & 34 & 14,9 & 6 & 60,5 & 49 & 199 & 19 & 104 & 31 & 1,22 & 1 & 10,3 & 31 & 0,66 & 49 & 220 & 34 \\
\hline 94 HT 31 QPM .......... & 7.059 & 48 & 15,0 & 9 & 57,2 & 7 & 201 & 25 & 102 & 23 & 1,05 & 18 & 13,0 & 44 & 0,76 & 46 & 220 & 35 \\
\hline 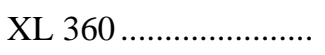 & 8.141 & 17 & 15,6 & 21 & 58,4 & 26 & 205 & 33 & 108 & 41 & 1,12 & 3 & 9,7 & 39 & 0,79 & 41 & 221 & 36 \\
\hline 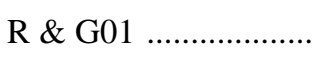 & 7.315 & 45 & 16,0 & 39 & 59,1 & 44 & 197 & 12 & 102 & 22 & 1,03 & 27 & 9,3 & 36 & 0,94 & 8 & 233 & 41 \\
\hline HATÃ $3012 \ldots \ldots \ldots \ldots . . . . .$. & 7.596 & 33 & 16,1 & 44 & 58,6 & 33 & 195 & 6 & 95 & 3 & 1,01 & 39 & 13,3 & 46 & 0,85 & 31 & 235 & 42 \\
\hline AGROMEN 2005 ..... & 8.253 & 12 & 15,9 & 38 & 58,6 & 36 & 205 & 34 & 106 & 38 & 1,02 & 35 & 8,8 & 25 & 0,88 & 24 & 242 & 43 \\
\hline 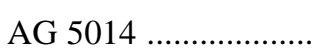 & 7.741 & 29 & 16,1 & 43 & 58,5 & 29 & 197 & 11 & 102 & 24 & 1,03 & 33 & 14,5 & 41 & 0,80 & 38 & 248 & 44 \\
\hline EXCELLER .................. & 7.544 & 35 & 15,9 & 33 & 58,7 & 38 & 210 & 42 & 105 & 35 & 1,05 & 20 & 8,4 & 22 & 0,87 & 25 & 250 & 45 \\
\hline 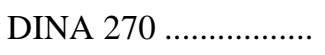 & 8.164 & 15 & 15,0 & 8 & 59,8 & 47 & 230 & 49 & 127 & 49 & 1,00 & 46 & 9,1 & 29 & 0,93 & 11 & 254 & 46 \\
\hline HD 9486 ......................... & 7.858 & 26 & 15,6 & 16 & 59,1 & 43 & 211 & 44 & 107 & 39 & 1,04 & 26 & 8,6 & 33 & 0,78 & 42 & 269 & 47 \\
\hline AL 25 XII ..................... & 7.430 & 38 & 15,0 & 7 & 60,4 & 48 & 219 & 47 & 118 & 48 & 1,04 & 22 & 15,1 & 49 & 0,86 & 30 & 289 & 48 \\
\hline PL 320 & 7.335 & 43 & 15,8 & 31 & 59,2 & 45 & 221 & 48 & 114 & 47 & 1,03 & 29 & 13,7 & 42 & 0,93 & 12 & 297 & 49 \\
\hline
\end{tabular}


Por não fazer pressuposições sobre a existência de um valor genotípico populacional a ser melhorado, além de não necessitar estimativas de parâmetros genéticos, pode, ao menos teoricamente, ser usado para a seleção de cultivares.

Embora os dados tenham sido estandardizados, optou-se por apresentar os resultados com aqueles originais, incluindo seus números de ordem. Nenhum peso foi atribuído a caráter algum, embora isso seja possível para esse índice e ao de soma de classificação, sendo essa uma vantagem que apresentam sobre o índice multiplicativo. Os valores para a distância euclidiana variaram de 5,73 a 10,55, com média 7,48. Vale lembrar que o melhorista teria à disposição para praticar seleção apenas os valores da distância euclidiana, sem tomar conhecimento daqueles revelados pelos híbridos selecionados para todos os caracteres de interesse, não podendo julgar se a seleção foi ou não bem realizada. Observando-se os resultados, nota-se que entre os dez primeiros classificados estão aqueles com número $6,37,3,19,5,14$, 10, 20, 4 e 24, para PE, caráter mais importante. Desses materiais, com exceção talvez daqueles com classificação 3, 4, 5 e 6, os restantes, de modo algum, seriam recomendados, o que invalida tal índice para a seleção de cultivares. Além disso, não há como saber se as diferenças entre as médias usadas nos cálculos são diferentes entre si, como por exemplo, entre o $14 .^{\circ}$ e o 10. material quanto a PE (híbridos G 167 S e C 625). Esse tipo de dúvida dificulta seu uso na prática. Mesmo que se optasse por algum peso na expressão da distância euclidiana para $\mathrm{PE}$, mais importante, isso nem sempre é fácil de realizar (Quadro 3).

Os híbridos com o $1 .^{\circ}$ e o $2 .^{\circ}$ valor de PE posicionaram-se em $44 .^{\circ}$ lugar (C $505 \mathrm{C}$ ) e $26^{\circ}$ lugar (C 505 B) respectivamente, colocações não razoáveis para caráter tão importante. Desse modo, tal forma de agrupar os valores de todos os caracteres num índice não é adequada à seleção de cultivares. Outro problema nos resultados refere-se ao fato de o índice não permitir o descarte de genótipos que não satisfaçam às exigências mínimas do mercado. Será que, por exemplo, o híbrido localizado em 1. . lugar não apresenta $\mathrm{R}^{2}$ abaixo de um nível mínimo aceito comercialmente? A não-elucidação desse tipo de questão ao melhorista dificulta ainda mais o seu uso.

\subsection{Comparação dos índices}

Pelo exposto, ficou claro que nenhum dos índices não paramétricos avaliados é adequado à seleção de cultivares. Resta a dúvida se poderiam ser aplicados em programas de seleção recorrente. $\mathrm{O}$ único que buscou relacionar-se com os índices lineares, em que há uma boa base teórica garantindo-lhe a validade, foi o multiplicativo: este, contudo, apenas aceita a situação onde todos os caracteres têm a mesma massa, caso muito particular; pode ser que isso limite muito sua aplicação, mesmo para a seleção recorrente.

O índice de soma de classificação, teoricamente poderia ter uso em programas de seleção recorrente, em que eventuais valores desfavoráveis em alguns atributos podem ser compensados por outros caracteres em níveis melhores. Fica difícil, contudo, afirmar sua validade nesse caso, uma vez que a base teórica empregada em seu desenvolvimento não o relaciona com nenhum outro índice. Comparando-o com o multiplicativo, verifica-se que eles apresentam uma baixa correlação $(r=-0,61)$, negativa porque o índice multiplicativo toma os maiores valores e, o índice de soma de classificação, os menores; contudo, pode-se chegar a resultados diferentes quando eles são usados. Os cinco melhores híbridos selecionados com o índice multiplicativo são XL 345, Z 84 E 98, Z 8452, C 625 e HD 9563; já para o de soma de classificação, XL 345, C 625, Z 84 E 98, A 953 e AGX 5558. Apenas três deles seriam selecionados com os dois índices, mostrando que a correlação não tem grande valor preditivo.

O índice da distância ao ideótipo, com aplicação sugerida em programas de seleção recorrente, apresentou alta correlação negativa $(r=-0,83)$ com o índice multiplicativo, negativa em função do escalonamento, além de alta correlação positiva com o índice de soma de classificação $(r=0,78)$. Os cinco melhores híbridos selecionados com ele foram Z 84 E 98, HD 9563, Z 8452, XL 345 e AGX 5558, sendo que quatro deles também seriam selecionados com o índice multiplicativo, mostrando que a magnitude da correlação serve para realizar predições. 
Quadro 3. Índice da distância ao ideótipo $\left(\mathrm{d}_{\mathrm{jI}}\right)$ para os 49 híbridos com classificação para $\mathrm{d}_{\mathrm{jI}}$ e cada uma das variáveis: produção de espigas (PE), umidade (U), número de dias para florescimento (FLO), altura da planta (AP), altura da espiga (AE), prolificidade (PROL), número de plantas acamadas e quebradas (PAQ)

\begin{tabular}{|c|c|c|c|c|c|c|c|c|c|c|c|c|c|c|c|c|c|c|c|c|}
\hline \multirow[t]{2}{*}{ Híbrido } & \multicolumn{2}{|c|}{ PE } & \multicolumn{2}{|c|}{$\mathrm{U}$} & \multicolumn{2}{|c|}{ FLO } & \multicolumn{2}{|c|}{$\mathrm{AP}$} & \multicolumn{2}{|c|}{$\mathrm{AE}$} & \multicolumn{2}{|c|}{ PROL } & \multicolumn{2}{|c|}{ PAQ } & \multicolumn{2}{|c|}{$\mathrm{R}^{2}$} & \multicolumn{2}{|c|}{$\mathrm{b}$} & \multicolumn{2}{|c|}{$\mathrm{d}_{\mathrm{jI}}$} \\
\hline & kg.ha- ${ }^{-1}$ & & $\%$ & & dias & & $\mathrm{cm}$ & & $\mathrm{cm}$ & & & & $\%$ & & & & & & & \\
\hline HD 9563 & 7.512 & 37 & 15,0 & 40 & 58,6 & 15 & 202 & 22 & 101 & 31 & 1,13 & 2 & 6,3 & 36 & 0,91 & 17 & 1,00 & 24 & 5,81 & 2 \\
\hline Z 8452 & 8.998 & 3 & 15,7 & 26 & 58,9 & 9 & 201 & 27 & 103 & 24 & 1,07 & 9 & 3,3 & 48 & 0,85 & 32 & 1,05 & 19 & 5,86 & 3 \\
\hline XL 345 & 8.096 & 19 & 15,6 & 32 & 56,7 & 49 & 197 & 40 & 98 & 40 & 1,06 & 13 & 6,9 & 29 & 0,91 & 15 & 0,81 & 44 & 6,05 & 4 \\
\hline C 625 & 8.212 & 14 & 15,9 & 16 & 57,6 & 39 & 197 & 41 & 95 & 46 & 1,02 & 34 & 4,9 & 44 & 0,95 & 6 & 0,90 & 35 & 6,30 & 6 \\
\hline G $167 \mathrm{~S}$ & 8.337 & 10 & 15,8 & 18 & 58,3 & 25 & 203 & 20 & 103 & 22 & 1,07 & 8 & 5,6 & 43 & 0,79 & 40 & 1,06 & 16 & 6,34 & 7 \\
\hline C $435 \mathrm{G}$ & 8.061 & 20 & 15,6 & 30 & 58,1 & 31 & 209 & 10 & 106 & 13 & 1,06 & 10 & 3,4 & 45 & 0,91 & 16 & 1,08 & 14 & 6,43 & 8 \\
\hline P 3041 & 8.596 & 4 & 15,9 & 12 & 58,2 & 27 & 208 & 11 & 105 & 16 & 1,05 & 16 & 5,2 & 40 & 0,86 & 29 & 0,98 & 26 & 6,54 & 9 \\
\hline AGX 5542 & 7.942 & 24 & 15,7 & 28 & 58,7 & 13 & 196 & 42 & 101 & 33 & 1,06 & 12 & 11,9 & 13 & 0,90 & 18 & 1,14 & 11 & 6,64 & 10 \\
\hline DINA 657 & 8.155 & 16 & 16,0 & 10 & 58,9 & 10 & 200 & 28 & 105 & 17 & 1,05 & 17 & 7,9 & 31 & 0,92 & 14 & 0,89 & 36 & 7,02 & 15 \\
\hline G $176 \mathrm{~S}$ & 8.453 & 7 & 16,1 & 8 & 57,6 & 38 & 193 & 47 & 96 & 45 & 1,00 & 42 & 11,3 & 10 & 0,96 & 4 & 1,05 & 17 & 7,04 & 16 \\
\hline Z 64 E 74 & 8.002 & 22 & 15,7 & 23 & 56,8 & 48 & 198 & 35 & 96 & 44 & 1,00 & 44 & 12,5 & 7 & 0,93 & 10 & 1,04 & 21 & 7,20 & 17 \\
\hline AGROMEN 2007 & 8.240 & 13 & 15,2 & 38 & 58,1 & 30 & 211 & 7 & 108 & 10 & 1,04 & 25 & 7,1 & 35 & 0,77 & 45 & 1,12 & 12 & 7,21 & 18 \\
\hline C $491 \mathrm{~W}$ & 7.519 & 36 & 15,9 & 14 & 58,0 & 32 & 204 & 19 & 101 & 32 & 1,08 & 6 & 15,5 & 3 & 0,93 & 13 & 1,14 & 10 & 7,22 & 19 \\
\hline AGROMEN 2018 & 7.649 & 31 & 15,7 & 25 & 57,0 & 45 & 197 & 36 & 103 & 25 & 1,03 & 28 & 9,6 & 18 & 0,90 & 21 & 0,77 & 46 & 7,30 & 20 \\
\hline DINA 1000 & 8.130 & 18 & 16,6 & 3 & 58,9 & 8 & 199 & 32 & 104 & 18 & 1,04 & 23 & 5,3 & 39 & 0,95 & 7 & 0,95 & 29 & 7,33 & 21 \\
\hline AS 3466 & 7.600 & 32 & 16,0 & 9 & 58,0 & 33 & 190 & 49 & 96 & 43 & 1,03 & 31 & 5,8 & 37 & 0,82 & 37 & 0,85 & 41 & 7,35 & 22 \\
\hline Z 84 E 90 & 7.430 & 39 & 15,9 & 15 & 57,4 & 41 & 194 & 46 & 94 & 48 & 1,00 & 40 & 8,4 & 26 & 0,94 & 9 & 1,00 & 22 & 7,38 & 23 \\
\hline A 953 & 6.843 & 49 & 14,5 & 49 & 56,9 & 47 & 198 & 34 & 100 & 35 & 1,06 & 14 & 4,0 & 47 & 0,77 & 44 & 0,83 & 42 & 7,39 & 24 \\
\hline
\end{tabular}




\begin{tabular}{|c|c|c|c|c|c|c|c|c|c|c|c|c|c|c|c|c|c|c|c|c|}
\hline \multirow[t]{2}{*}{ Híbrido } & \multicolumn{2}{|c|}{$\mathrm{PE}$} & \multicolumn{2}{|c|}{$\mathrm{U}$} & \multicolumn{2}{|c|}{ FLO } & \multicolumn{2}{|c|}{$\mathrm{AP}$} & \multicolumn{2}{|c|}{$\mathrm{AE}$} & \multicolumn{2}{|c|}{ PROL } & \multicolumn{2}{|c|}{ PAQ } & \multicolumn{2}{|c|}{$\mathrm{R}^{2}$} & \multicolumn{2}{|c|}{$\mathrm{b}$} & \multicolumn{2}{|l|}{$\mathrm{d}_{\mathrm{jI}}$} \\
\hline & kg.ha-1 & & $\%$ & & dias & & $\mathrm{cm}$ & & $\mathrm{cm}$ & & & & $\%$ & & & & & & & \\
\hline C 505 B & 9.057 & 2 & 16,6 & 2 & 58,5 & 20 & 207 & 12 & 111 & 6 & 1,06 & 15 & 9,7 & 16 & 0,90 & 20 & 1,24 & 4 & 7,47 & 26 \\
\hline C 435 & 7.955 & 23 & 15,6 & 31 & 58,5 & 18 & 210 & 9 & 110 & 8 & 1,05 & 21 & 9,6 & 27 & 0,88 & 23 & 0,98 & 27 & 7,48 & 27 \\
\hline AGROMEN 2005 & 8.253 & 12 & 15,9 & 13 & 58,6 & 14 & 205 & 16 & 106 & 12 & 1,02 & 35 & 8,8 & 25 & 0,88 & 24 & 0,89 & 37 & 7,57 & 28 \\
\hline $\mathrm{CO} 36$ & 7.326 & 44 & 15,5 & 36 & 57,1 & 44 & 199 & 30 & 98 & 41 & 1,00 & 45 & 3,5 & 49 & 0,87 & 28 & 0,75 & 48 & 7,71 & 31 \\
\hline MASTER & 7.650 & 30 & 16,3 & 4 & 57,8 & 36 & 207 & 13 & 113 & 5 & 1,08 & 5 & 6,9 & 34 & 0,84 & 34 & 0,87 & 38 & 7,76 & 32 \\
\hline HT 951005 & 7.571 & 34 & 14,9 & 44 & 60,5 & 1 & 199 & 31 & 104 & 19 & 1,22 & 1 & 10,3 & 19 & 0,66 & 49 & 1,08 & 13 & 7,82 & 33 \\
\hline EXCELLER & 7.544 & 35 & 15,9 & 17 & 58,7 & 12 & 210 & 8 & 105 & 15 & 1,05 & 20 & 8,4 & 28 & 0,87 & 25 & 0,93 & 31 & $\mathbf{7 , 8 6}$ & 34 \\
\hline $\mathrm{CO} 34$ & 7.381 & 42 & 15,7 & 22 & 58,3 & 26 & 203 & 21 & 99 & 38 & 1,00 & 41 & 4,9 & 41 & 0,90 & 19 & 0,81 & 43 & $\mathbf{7 , 8 7}$ & 35 \\
\hline $\mathrm{R} \& \mathrm{G} 01$ & 7.315 & 45 & 16,0 & 11 & 59,1 & 6 & 197 & 38 & 102 & 28 & 1,03 & 27 & 9,3 & 14 & 0,94 & 8 & 0,92 & 34 & 8,09 & 39 \\
\hline Z 8440 & 7.264 & 47 & 15,3 & 37 & 56,9 & 46 & 193 & 48 & 91 & 49 & 1,00 & 43 & 9,5 & 15 & 0,77 & 43 & 0,77 & 47 & 8,11 & 40 \\
\hline AGX 5674 & 8.020 & 21 & 15,7 & 24 & 58,2 & 28 & 195 & 45 & 98 & 42 & 1,02 & 37 & 14,8 & 5 & 0,70 & 47 & 0,92 & 33 & 8,13 & 41 \\
\hline HD 9486 & 7.858 & 26 & 15,6 & 34 & 59,1 & 7 & 211 & 6 & 107 & 11 & 1,04 & 26 & 8,6 & 17 & 0,78 & 42 & 0,98 & 25 & 8,15 & 42 \\
\hline HATÃ 3012 & 7.596 & 33 & 16,1 & 6 & 58,6 & 17 & 195 & 44 & 95 & 47 & 1,01 & 39 & 13,3 & 4 & 0,85 & 31 & 1,21 & 5 & 8,24 & 43 \\
\hline C 505 C & 9.531 & 1 & 17,4 & 1 & 58,6 & 16 & 212 & 5 & 110 & 7 & 1,07 & 7 & 8,4 & 23 & 0,96 & 5 & 1,39 & 1 & 8,24 & 44 \\
\hline PL 322 & 7.313 & 46 & 15,6 & 33 & 57,4 & 42 & 216 & 4 & 114 & 4 & 1,02 & 36 & 7,2 & 30 & 0,97 & 1 & 1,19 & 6 & 8,41 & 45 \\
\hline FT 9006 & 7.383 & 41 & 15,0 & 39 & 58,4 & 23 & 196 & 43 & 100 & 36 & 1,01 & 38 & 15,0 & 2 & 0,83 & 35 & 0,86 & 40 & 8,54 & 46 \\
\hline PL 320 & 7.335 & 43 & 15,8 & 19 & 59,2 & 5 & 221 & 2 & 114 & 3 & 1,03 & 29 & 13,7 & 8 & 0,93 & 12 & 1,37 & 2 & $\mathbf{9 , 5 2}$ & 47 \\
\hline AL 25 XII & 7.430 & 38 & 15,0 & 43 & 60,4 & 2 & 219 & 3 & 118 & 2 & 1,04 & 22 & 15,1 & 1 & 0,86 & 30 & 0,94 & 30 & 10,30 & 48 \\
\hline DINA 270 & 8.164 & 15 & 15,0 & 42 & 59,8 & 3 & 230 & 1 & 127 & 1 & 1,00 & 46 & 9,1 & 21 & 0,93 & 11 & 1,18 & 8 & 10,55 & 49 \\
\hline
\end{tabular}


Já com o índice de soma de classificação, somente três seriam selecionados em comum. Isso é conseqüência da maior correlação entre o índice multiplicativo e o de distância ao ideótipo, mais semelhantes entre si. O índice de soma de classificação deve envolver princípios diferentes em seu desenvolvimento, levantando a dúvida sobre sua validade mesmo em programas de seleção recorrente, dada a diferença que apresenta em relação aos demais.

Para que os índices sejam realmente adequados à seleção recorrente, de algum modo a correlação genética entre os caracteres deveria ser considerada, uma vez que se está buscando o melhoramento de uma população como um todo. De forma geral, um índice de seleção deve permitir a classificação correta dos genótipos, considerando diversos caracteres simultaneamente. Tal classificação deve ser bem feita, para que se efetue a seleção dos genótipos superiores para um conjunto de caracteres. Na seleção recorrente, os genótipos selecionados dificilmente mostrarão bons valores para todos os caracteres, invalidando o uso de índices adequados a tal situação para a seleção de cultivares. O único desses índices que tem um princípio de desenvolvimento semelhante aos dos índices específicos para seleção recorrente é o multiplicativo. Como ele se correlacionou melhor apenas com o da distância ao ideótipo, eventualmente apenas esses dois podem efetivamente ser aplicados à seleção recorrente. Já para a seleção de cultivares, a intensidade de seleção aplicada é muito elevada, e como existem limitações sobre os níveis mínimos dos caracteres, um índice de seleção deve ser especificamente desenvolvido, ficando evidente que nenhum é adequado.

\section{CONCLUSÃO}

Nenhum dos três índices não paramétricos revistos se mostrou adequado à seleção de cultivares.

\section{REFERÊNCIAS BIBLIOGRÁFICAS}

BRIM, C.A.; JOHNSON, H.W. \& COCKERHAM, C.C. Multiple selection criteria in soybeans. Agronomy Journal, Madison, 51:42-46, 1959.

COCHRAN, W.G. \& COX, G.M. Experimental designs. New York, John Wiley, 1957. 617p.

EBERHART, S.A. \& RUSSELL, W.A. Stability parameters for comparing varieties. Crop Science, Madison, 6:3640, 1966.

ELSTON, R.C. A weight free index for the purpose of ranking of selection with respect to several traits at a time. Biometrics, Alexandria, 19:85-97, 1963.

FISHER, R.A. The use of multiple measurements in taxonomic problems. Annals Eugenics, 7:179-189, 1936.

KEMPTHORNE, O. \& NORDSKOG, A.W. Restricted selection indices. Biometrics, Alexandria, 15:10-19, 1959.

MULAMBA, N.N. \& MOCK, J.J. Improvement of yield potential of the Eto Blanco maize (Zea mays L.) population by breeding for plant traits. Egyptian Journal of Genetic and Cytology, Giza, 7:40-51, 1978.

PESEK, J. \& BAKER, R.J. Desired improvement in relation to selection indices. Canadian Journal of Plant Science, Otawa, 49:803-804, 1969.

SAS INSTITUTE INC. Statistical Analysis System. Release 6.08. (software). Cary, 1992.

SMITH, H.F. A discriminant function for plant selection. Annals Eugenics, 7:240-250, 1936.

SMITH, O.S.; HALLAUER, A.R. \& RUSSELL, W.A. Use of index selection in recurrent selection programs in maize. Euphytica, Dordrecht, 30:611-618, 1981.

STEEL, R.G.D. \& TORRIE, J.H. Principles and procedures of statistics. New York, McGraw Hill, 1960. 481p.

TAI, G.C.C. Index selection with desired gains. Crop Science, Madison, 17:182-183, 1977.

WRICKE, G. \& WEBER, W.E. Quantitative genetics and selection in plant breeding. New York, Walter de Gruyter, 1986. 406p. 